\title{
Dark Matter Annihilation to Neutrinos: Current Limits and Future Prospects
}

\section{Carlos A. Argüelles, ${ }^{a}$ Alejandro Diaz, ${ }^{b}$ Ali Kheirandish, ${ }^{c, *}$ Ibrahim Safa ${ }^{d, a}$ and Aaron C. Vincent ${ }^{e, f, g}$}

${ }^{a}$ Dept. of Physics \& Laboratory for Particle Physics \& Cosmology, Harvard University, Cambridge, MA 02138, USA

${ }^{b}$ Dept. of Physics, Massachusetts Institute of Technology, Cambridge, MA 02139 USA

${ }^{c}$ Dept. of Physics \& Center for Multimessenger Astrophysics Institute for Gravitation \& the Cosmos, The Pennsylvania State University, University Park PA 16802 USA

${ }^{d}$ Dept. of Physics \& Wisconsin IceCube Particle Astrophysics Center, University of Wisconsin, Madison, WI 53706, USA

${ }^{e}$ Dept.of Physics, Engineering Physics \& Astronomy, Queen's University, Kingston, ON K7L 3N6, Canada ${ }^{f}$ Arthur B. McDonald Canadian Astroparticle Physics Research Institute, Kingston, ON K7L 3N6, Canada ${ }^{g}$ Perimeter Institute for Theoretical Physics, Waterloo, ON N2L 2Y5, Canada

E-mail: kheirandish@psu.edu

\begin{abstract}
Neutrinos can escape dense environments, otherwise opaque to photons, and travel cosmic distances unscathed by background radiation or magnetic fields. While ideal cosmic messengers, they present a unique opportunity to test physics beyond the Standard Model, especially dark matter. Moreover, there is a distinct possibility that the neutrino sector is the principal portal through which the dark matter interacts with the Standard Model. In this talk, we will discuss new opportunities offered by high-energy neutrinos and provide new model-independent limits on dark matter annihilation into neutrinos based on measurements of neutrinos. We present the most up-to-date and comprehensive results on dark matter annihilation into neutrinos using the most recently available data from neutrino telescopes, with measurements spanning a wide energy range. Finally, we will present the projections for next-generation of neutrino experiments.
\end{abstract}

$37^{\text {th }}$ International Cosmic Ray Conference (ICRC 2021)

July 12 th - 23rd, 2021

Online - Berlin, Germany

\footnotetext{
*Presenter
} 


\section{Introduction}

Weakly interacting massive particles (WIMPs) are the primary candidates for particle dark matter (DM). The WIMP hypothesis yields a thermally average cross-section rate, $\langle\sigma v\rangle \simeq 3 \times$ $10^{-26} \mathrm{~cm}^{3} / \mathrm{s}$, which can explain the observed relic abundance after the freeze-out and is independent of the annihilation products. Meanwhile, thermal production of WIMPs in the early Universe implies possible ongoing annihilation of DM to Standard Model (SM) particles. This possibility has facilitated the indirect search for dark matter.

In this study, we focus on the direct annihilation of DM to neutrinos. This presents the most invisible channel for DM annihilation search. The distinct possibility that neutrinos might be the principal portal to the dark sector facilitates this assumption. Such possibility is motivated by the scotogenic models where neutrinos' mass is achieved via interaction with DM, see e.g. [1]. Furthermore, the upper limit on the DM annihilation cross-section to neutrinos serves as an upper bound on DM annihilation to SM particles, as the latter is larger [2].

In general, DM could annihilate to all SM particles. Annihilation to most SM particles results in the production of gamma rays and neutrinos. Today, the most constraining limits on DM annihilation cross-section are obtained from the absence of signal in multiwavelength observations, especially from the Milky Way and its satellite galaxies. However, there is a distinct possibility for DM directly annihilating to neutrinos which makes indirect search via neutrinos important. Moreover, neutrinos, contrary to photons, can arrive from the edge of the Universe with negligible attenuation or obscuring. As such, we can search for heavy DM with neutrinos, which is not viable with electromagnetic radiation.

The global spectrum of neutrinos spans a wide range of energy, from relic cosmic neutrino background with energies of $\mathrm{eV}$ to long-sought neutrinos produced in the interaction of ultra-highenergy cosmic rays with the $\mathrm{CMB}$ with energies exceeding $10^{18} \mathrm{eV}$. Figure 1 shows the spectrum of neutrinos from the sky from low to high energies. The figure is a mixture of observations and predictions. For energies, $\gtrsim 0.1 \mathrm{MeV}$, there exist an interrupted flux of neutrinos from the sky. An extraordinary amount of data has been collected in this range for measuring neutrino fluxes which can be utilized to search for DM annihilation to neutrinos. In this proceeding, we summarize our recent work [3] on the compendium of constraints on DM annihilation to neutrinos. We present new limits whilst we provide previously reported constraints in a consistent way but adjusting the underlying assumptions with a unique set of DM parameters. We demonstrate that neutrino telescopes are closing in on the parameter space of DM annihilation. While for the lowmass regime, the limits are already approaching the relic density values, the current and upcoming neutrino telescopes aiming at very high energies are opening a new avenue for the indirect search of DM.

\section{Neutrino flux from dark matter annihilation}

In direct DM annihilation to neutrino-antineutrino pairs, a neutrino will carry energies equal to the DM mass. That is, $E_{v}=m_{\chi}$. The energy spectrum of neutrinos is therefore

$$
\frac{d N_{v}}{d E_{v}}=2 \delta\left(1-\frac{E_{v}}{m_{\chi}}\right) \frac{m_{\chi}}{E_{v}^{2}}
$$




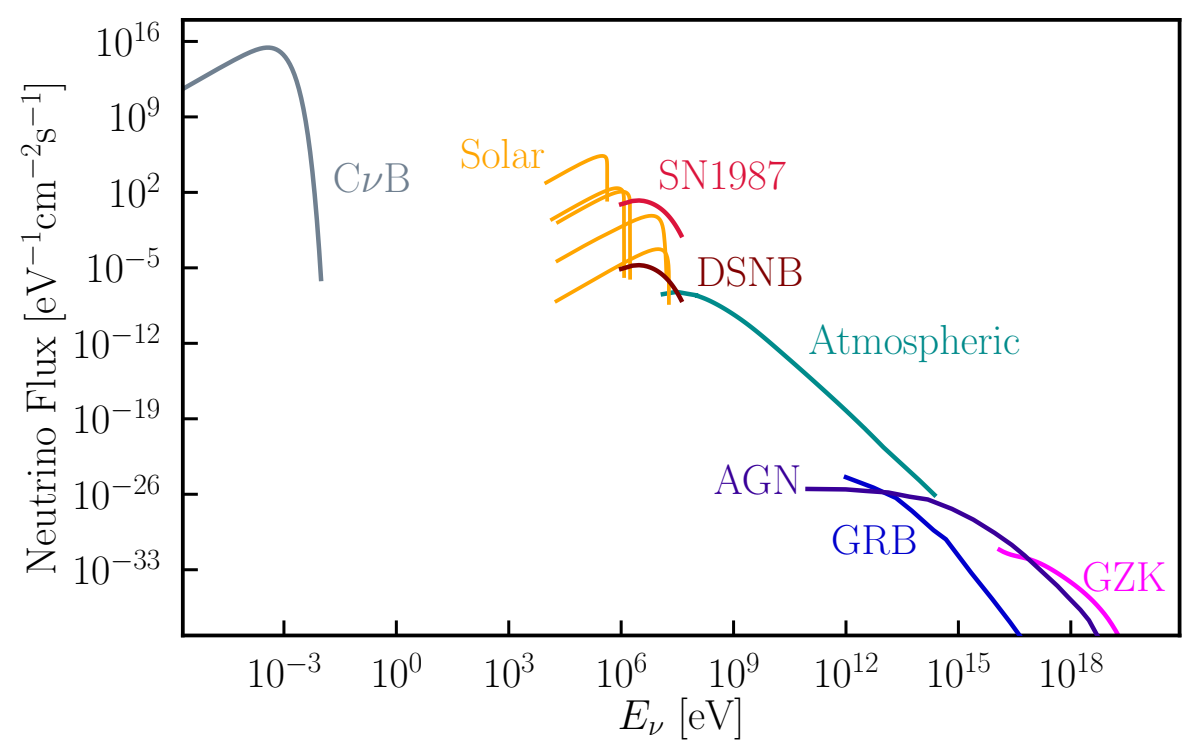

Figure 1: The cosmic-neutrino spectrum. Sources are the Big Bang $(\mathrm{C} v \mathrm{~B})$, the Sun, supernovae $(\mathrm{SN})$, atmospheric neutrinos, active galactic nuclei (AGN) galaxies, and GZK neutrinos.

at the site where annihilation is occurring. There are two main contributions to the neutrino flux: Galactic and extragalactic. The former contribution comes from DM annihilation to neutrinos in the Milky Way and the DM halo surrounding it. This contribution provides an anisotropic neutrino emission that peaks around the center of the Galaxy. The extragalactic signal includes neutrino emission from structures beyond the Milky Way and is an integral over all redshifts. The resulting neutrino emission is therefore isotropic.

Assuming equal flavor composition, the Galactic contribution to the neutrino flux per flavor is given by

$$
\frac{d \Phi_{v+\bar{v}}}{d E_{v}}=\frac{1}{4 \pi} \frac{\sigma v}{\kappa m_{\chi}^{2}} \frac{1}{3} \frac{d N_{v}}{d E_{v}} J(\Omega)
$$

where $\kappa=2$ (4) for Majorana (Dirac) DM. In this study, we set $\kappa=2$ (Majorana DM). $m_{\chi}$ is the DM mass and $J(\Omega)$ is a three-dimensional integral over the target solid angle in the sky, $d \Omega$, and the distance $d x$ along the line of sight (l.o.s.) of the DM density $\rho_{\chi}$, namely

$$
J \equiv \int d \Omega \int_{\text {l.o.s. }} \rho_{\chi}^{2}(x) d x .
$$

We use a generalized NFW profile to describe the DM halo, given by

$$
\rho_{\chi}(r)=\rho_{s} \frac{2^{3-\gamma}}{\left(\frac{r}{r_{s}}\right)^{\gamma}\left(1+\frac{r}{r_{s}}\right)^{3-\gamma}} .
$$

We use DM halo parameters compatible with the best-fit values of [4]. When calculating the $J$-factor, we take into account the effective coverage of the neutrino detectors. Some experiments, such as IceCube, have an all-sky coverage. However, experiments like ANITA and GRAND only access certain parts of the sky. For these experiments, we recompute the J-factor by adapting their 


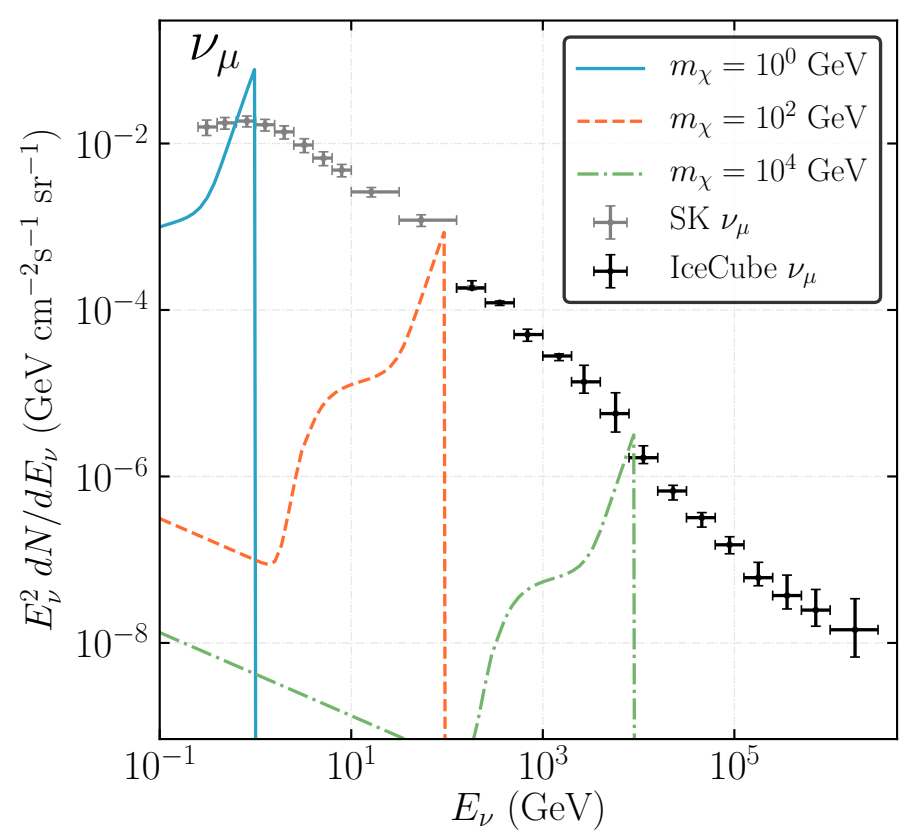

Figure 2: An Example of neutrino fluxes produced by DM annihilation overlayed on the observed neutrino flux measurement. Expected flux of neutrinos from extragalactic DM annihilation as a function of energy, shown for several DM masses. Here, the extragalactic DM annihilation fluxes are compared to the unfolded muon neutrino atmospheric fluxes from both Super-Kamiokande [5] and IceCube [6, 7].

respective sensitivity, and from local coordinates to Galactic coordinates and integrate over the accessible region of the sky for each experiment in Galactic coordinates.

The isotropic extragalactic contribution has two components: flux from the non-collapsed distribution of DM and contribution from overdensities in galactic halos across the Universe. The expected neutrino flux, therefore, is given by

$$
\frac{d \Phi_{\nu+\bar{\nu}}}{d E_{v}}=\frac{1}{4 \pi} \frac{\Omega_{D M}^{2} \rho_{c}^{2} \sigma_{v}}{\kappa m_{x}^{2}} \frac{1}{3} \int_{0}^{z_{u p}} d z \frac{(1+G(z))(1+z)^{3}}{H(z)} \frac{d N_{v+\bar{\nu}}\left(E^{\prime}\right)}{d E^{\prime}},
$$

where $d N_{v}\left(E^{\prime}\right) / d E$ is the neutrino energy spectrum at the Earth, where $E^{\prime}(E)$ is the energy at the source (detector). The source production spectrum in Eq. 1 is modified with the redshift as

$$
\frac{d N_{\nu+\bar{\nu}}\left(E^{\prime}\right)}{d E^{\prime}}=2 \frac{m_{\chi}}{E^{\prime 2}} \delta\left(\frac{m_{\chi}}{E^{\prime}}-1\right)=\frac{2}{E} \delta\left[z-\left(\frac{m_{\chi}}{E}-1\right)\right] .
$$

In Eq. 5, the first part of the factor $1+G(z)$ in the integrand represents the isotropic background DM contribution, while $G(z)$ is the halo boost factor at redshift $z$, which accounts for the enhancement to the annihilation rate in DM clusters, as well as their evolution with redshift.

In Fig. 2, we showcase the neutrino spectrum of DM annihilation to neutrino-antineutrino pairs from cosmological sources. We overlay the measured muon neutrino flux in Super Kamiokande (SK) and IceCube. Fluxes are computed using the value of the cross-section corresponding to the $90 \%$ C.L. limit derived in this work. 


\section{Upper Limits on Dark Matter Annihilation to Neutrinos}

So far, no observation has found a signal from DM annihilation leading to constraints on the annihilation of DM to neutrinos in a variety of experiments, each aiming at a specific range of energies. These measurements include the observed solar, atmospheric, and astrophysical neutrino fluxes, as well as upper limits on the diffuse supernova background and GZK neutrinos. We utilize the reported neutrino flux to deduce the upper limit on the DM annihilation cross-section rate. In order to find the upper limit on $\langle\sigma v\rangle$, we estimate the expected signal and background. We estimate the signal flux from Eqs. 2 and 5. The background in each energy range depends on the origin of the flux and characteristics of particle detection in each detector. The upper limits provided here also depend largely on the systematics that govern the neutrino detection in each range or detection technique.

In order to find the upper limit, we perform a likelihood analysis that incorporates this information where possible. In Fig. 3, we present the upper limits on DM annihilation cross-section from sub-GeV DM to superheavy DM with $m_{\chi}$ exceeding PeVs. The lines marked with a heart show the limits obtained in our study. The rest of the lines are complied from measurements of experimental collaboration or independent groups. In order to make a uniform comparison, when possible, we have re-scaled the $J$-factor for the limits or the sensitivities of the experiments.

At $m_{\chi} \sim \mathrm{MeV}$, limits obtained from upper limits from Borexino [8] and KAMLAND [9] constrain the DM cross-section rate better than $10^{-21} \mathrm{~cm}^{3} / \mathrm{s}$. At slightly higher energies, SK dominates the upper limit, especially the upper limits found from the search for diffuse supernova background [10] is strong and touches the thermal relic line. We also show the limit from [11] that limits DM cross-section for masses below $\mathrm{GeV}$. Around $\mathrm{GeV}$ mass, the dominant neutrino spectrum at Earth is the atmospheric neutrinos, produced by very high-energy cosmic-ray interaction. SK and IceCube provide a precise measurement of this flux. Here, we estimated the upper limits from SK measurement at lower energies as well as the limits reported by the SK $[12,13]$ and IceCube [14] Collaborations. Above $100 \mathrm{GeV}$, ANTARES presents the most constraining limit until $m_{\chi} \sim 10^{5} \mathrm{GeV}$ [15]. We show the limits obtained from IceCube high-energy neutrino flux measurement [16] in the same energy range, which extends to $m_{\chi} \sim 10 \mathrm{PeV}$. The strongest limits are from analyses that incorporate both energy and direction for the neutrinos.

At the highest energies, in Fig. 3, we show the limit from IceCube EHE analysis [17]. The limit is deduced from the upper limit on the cosmogenic neutrino flux in 9 years of IceCube data. These limits assume $100 \%$ of the dark matter is composed of a given Majorana particle. If instead only a fraction, $f$, is considered, these results should be multiplied by $1 / f^{2}$. In the case of Dirac DM, limits would be scaled up by a factor of two.

In addition to the upper limits from the current neutrino experiments, we provide the prospects for studying DM annihilation to neutrinos from future neutrino experiments. At low energies, DUNE and Hyper Kamiokande are going to establish a strong measurement of the annihilation crosssection, narrowing down the allowed parameter space. At higher energies, utilizing atmospheric and IceCube astrophysical neutrinos, the sensitivity would be improved the development detector in the Mediterranean, KM3NeT, and in Lake Baikal, Baikal GVD. Neutrino telescopes' reach to ultrahigh-energies is going to be enhanced by the development and commissioning of P-One, IceCube Gen-2, and GRAND. These planned neutrino telescopes will establish a survey of DM annihilation 


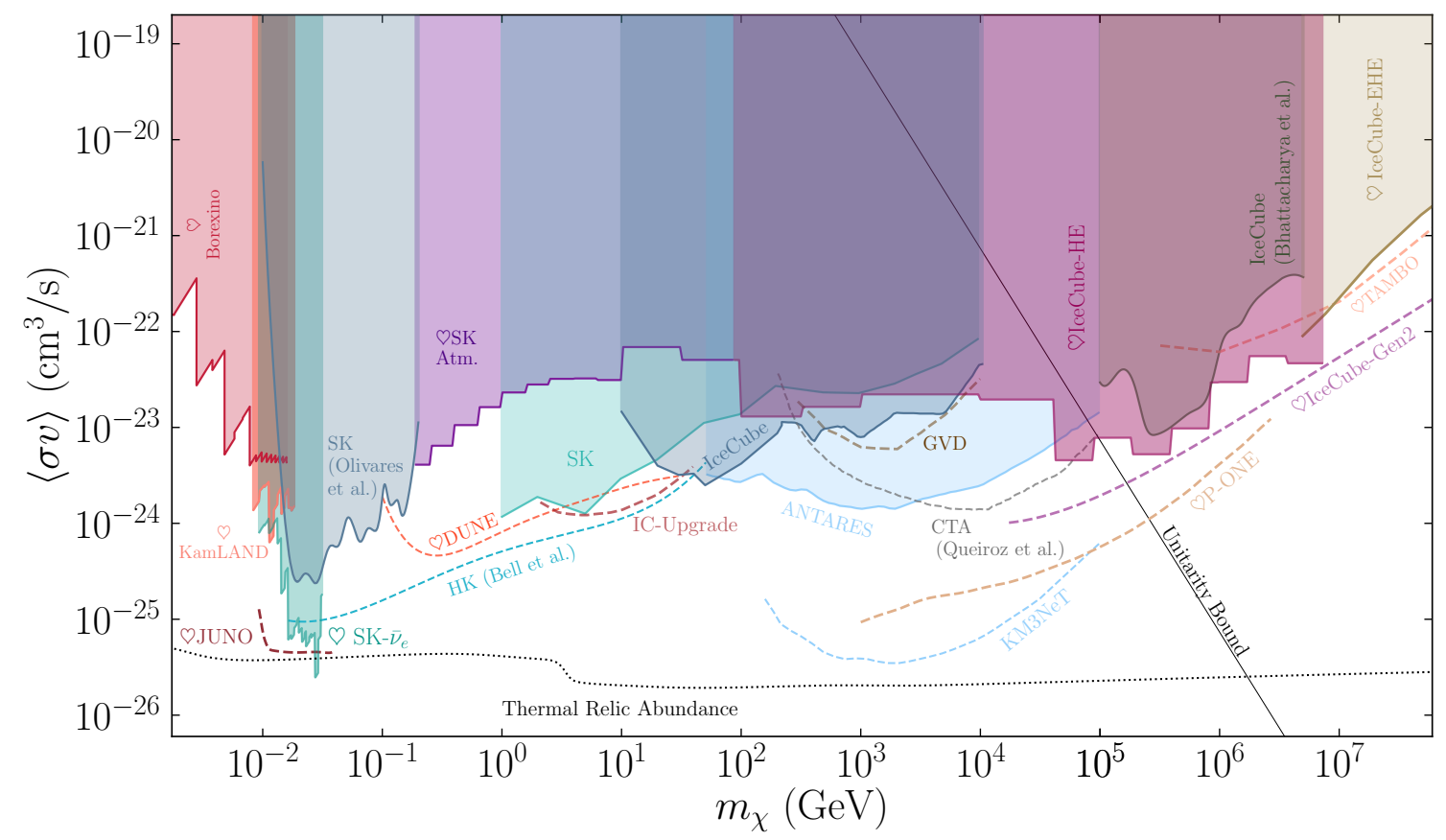

Figure 3: The landscape of DM annihilation into neutrinos up to $10^{8} \mathrm{GeV}$. We show new limits, as well as previously published limits. Solid and dashed lines represent 90\% C.L. limits and sensitivities, respectively. Projected sensitivities assume 5 years of data taking for neutrino experiments and 100 hours of observation for CTA. The dotted line corresponds to the value required to explain the observed abundance via thermal freeze-out. The straight diagonal line, labeled as "Unitarity Bound," gives the maximum allowed cross section for a non-composite DM particle. These results assume $100 \%$ of the dark matter is composed of a given Majorana particle. See Fig. 4 for constraints and projections up to $10^{11} \mathrm{GeV}$.

for very heavy DM and surpass the region that is constrained by the Unitarity of the cross-section.

Figure 4 presents a close up of the limits for heavy DM with $m_{\chi}>\mathrm{TeV}$. In addition to the limit from IceCube-EHE analysis, we also show the upper limit from Auger cosmogenic neutrino search [18]. Future neutrino telescopes aiming at measuring neutrino flux beyond $\mathrm{PeV}$ energies, such as IceCube Gen-2, GRAND, and RNO-G are going to further improve sensitivity for superheavy DM searches.

The limits we present here are for the s-wave, where DM annihilation cross-section is not velocity dependent. For p- and d-wave limits, see [3].

\section{Summary and Outlook}

Thanks to the experimental advances in neutrino detection, we are able to obtain a comprehensive set of limits on DM annihilation directly to neutrino-antineutrino pairs, for a DM mass range spanning 15 orders of magnitude; from $10^{-3} \mathrm{GeV}$ to $10^{12} \mathrm{GeV}$. This energy range is remarkably covered by a variety of neutrino experiments searching for neutrinos from supernova background, sun, atmosphere, and the sources of IceCube high-energy neutrinos. In this landscape, the strongest limits are obtained from SK, ANTARES, and IceCube. Naturally, analyses that utilize the arrival direction of neutrinos, in addition to their energy, are more sensitive. 


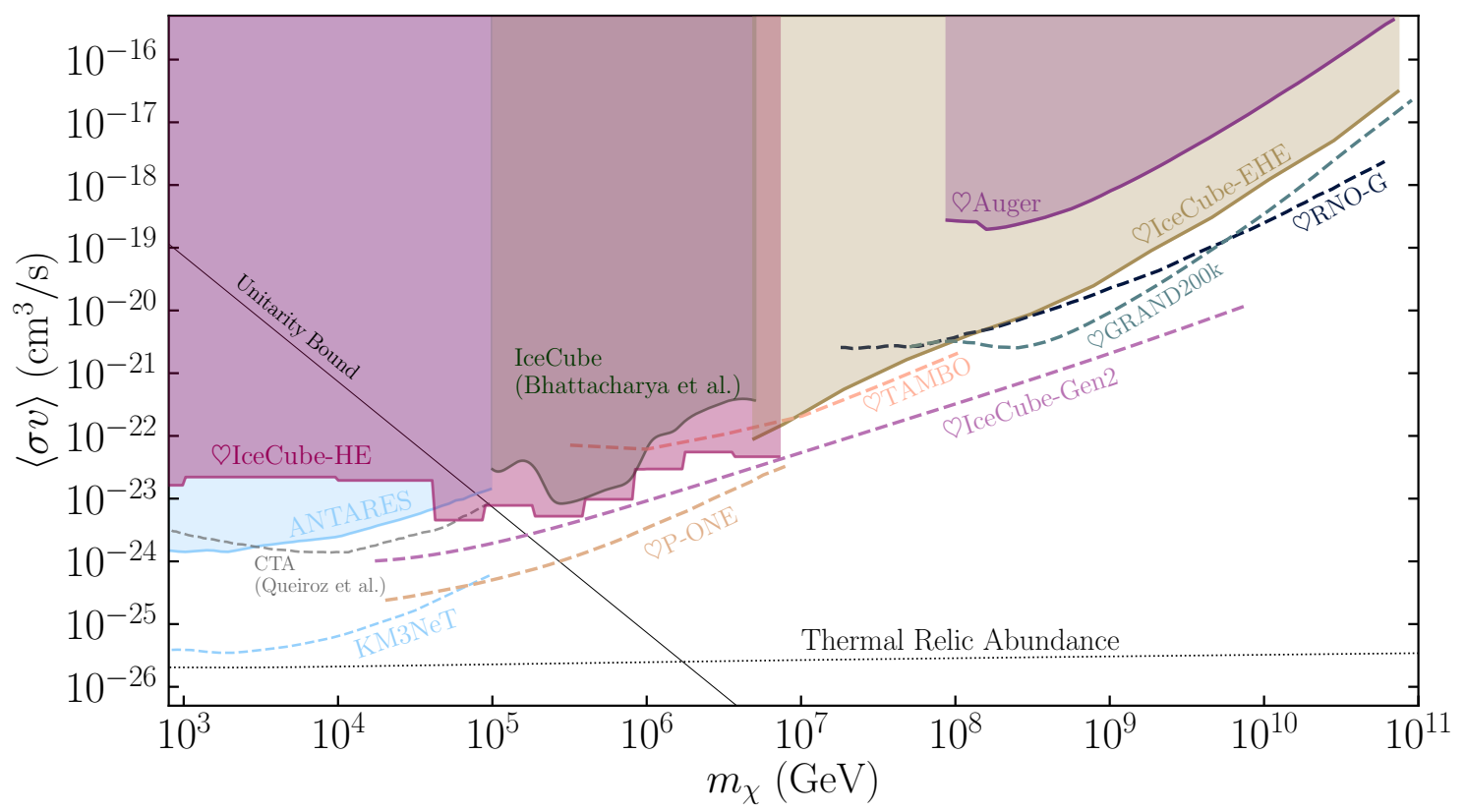

Figure 4: The landscape of supra-TeV dark matter annihilation into neutrinos. Same as Fig. 3, but for the high-mass region. All the experimental constraints in this plot are calculated by converting either the detected flux or the reported upper limit into a conservative upper bound on the DM annihilation cross section.

As the DM mass increases, the annihilation rate would decreases as the DM density is unchanged. This effect is visible at the very high energy region of Figs 3 and 4. However, remarkably, the upper limit and sensitivity to the DM annihilation signal are nearly flat for $m_{\chi} \lesssim \mathrm{PeV}$, partially thanks to the growing size of the neutrino telescopes.

The sensitivity of the next generation of neutrino experiments will approach the expected thermal relic abundance. Especially around $10 \mathrm{MeV}$, the gap between the limits and the relic will narrow. For high energies, the commissioning of KM3NeT with cubic kilometer-scale volume will boost the sensitivity closer to the thermal relic line. At very high energies, where neutrinos become the only probe of the high-energy universe. The upcoming neutrino observatories will further improve the sensitivity to probe for superheavy DM.

Neutrinos were the first particle that insinuated the necessity of physics beyond the Standard Model. The uninterrupted measurement of the neutrino spectrum offers a unique opportunity to probe the most invisible channel of DM annihilation. The constraints presented here are narrowing down the parameter space for DM annihilation to standard model products. 


\section{References}

[1] C. Boehm, Y. Farzan, T. Hambye, S. Palomares-Ruiz, and S. Pascoli Phys. Rev. D77 (2008) 043516.

[2] J. F. Beacom and M. R. Vagins Phys. Rev. Lett. 93 (2004) 171101.

[3] C. A. Argüelles, A. Diaz, A. Kheirandish, A. Olivares-Del-Campo, I. Safa, and A. C. Vincent Review of Modern Physics $(12,2019)$.

[4] M. Benito, A. Cuoco, and F. Iocco JCAP 1903 no. 03, (2019) 033.

[5] Super-Kamiokande Collaboration, E. Richard et al. Phys. Rev. D94 no. 5, (2016) 052001.

[6] IceCube Collaboration, M. G. Aartsen et al. Phys. Rev. D91 (2015) 122004.

[7] IceCube Collaboration, M. G. Aartsen et al. Astrophys. J. 833 no. 1, (2016) 3.

[8] Borexino Collaboration, G. Bellini et al. Phys. Lett. B696 (2011) 191-196.

[9] KamLAND Collaboration, A. Gando et al. Astrophys. J. 745 (2012) 193.

[10] W. Linyan, Experimental Studies on Low Energy Electron Antineutrinos and Related Physics. PhD thesis, Tsinghua University, 2018. http:

//www-sk.icrr.u-tokyo.ac.jp/sk/_pdf/articles/2019/SKver-Linyan.pdf? fbclid=IwAR0EgdX5Clr1tFJI1tXV2W4YjnD12wfiyZ420naRGeJRnvJxGsUu6Mi--dA.

[11] A. Olivares-Del-Campo, Dark Matter and Neutrinos: A Love-Hate Relationship. PhD thesis, Durham U., 2019. http: //etheses . dur . ac . uk/13142/.

[12] Super-Kamiokande Collaboration, K. Frankiewicz, "Searching for Dark Matter Annihilation into Neutrinos with Super-Kamiokande," in Proceedings, Meeting of the APS Division of Particles and Fields (DPF 2015): Ann Arbor, Michigan, USA, 4-8 Aug 2015. 2015. arXiv: 1510.07999 [hep-ex].

[13] K. Frankiewicz, Indirect Search for Dark Matter with the Super-Kamiokande Detector. PhD thesis, National Centre for Nuclear Research, Poland, 2018.

[14] IceCube Collaboration, M. G. Aartsen et al. Eur. Phys. J. C76 no. 10, (2016) 531.

[15] KM3Net Collaboration, S. Adrian-Martinez et al. J. Phys. G43 no. 8, (2016) 084001.

[16] IceCube Collaboration, M. G. Aartsen et al. Eur. Phys. J. C77 no. 10, (2017) 692.

[17] IceCube Collaboration, M. G. Aartsen et al. Phys. Rev. D98 no. 6, (2018) 062003.

[18] Pierre Auger Collaboration, E. Zas PoS ICRC2017 (2018) 972. [,64(2017)]. 